\section{Historia de la escrófula: de la discrasia humoral a la consunción}

\author{
IGNACIO DUARTE G. ${ }^{1,2}$, CLAUDIA CHUAQUI F.,a
}

\section{History of scrofula: from humoral dyscrasia to consumption}

The term "scrofula" was used for a long time to designate a chronic swelling of cervical lymph nodes. This paper outlines the prevalent ideas on the nature, pathogenesis and the treatment of this disorder, from classical Greek medicine up to the $18^{\text {th }}$ century. A Hippocratic treatise regarded scrofula as produced by an accumulation of phlegm, with a consequent imbalance or dyscrasia of the body humors. It was believed that it could heal spontaneously; but it could also soften, open through the skin and have an obstinate course. The treatment consisted mainly on local applications, incision to evacuate the soft content, or extirpation of the abnormal mass. In France and England, crowds of scrofulous patients were touched by the kings who were supposed to have a hereditary miraculous power to cure the disease. A Medieval text mentioned that scrofula could also affect other parts of the body. In the 17th century, scrofula was reputed as a frequent condition and was attributed to blood acrimony which coagulated in spongy organs. It was associated to phthisis or consumption due to the lethal outcome in some patients and to a cheese-like appearance of the pulmonary and the scrofulous lesions.

(Rev Med Chile 2016; 144: 503-507)

Key words: Humoralism; King's evil; Mycobacterium tuberculosis; Mycobacterium scrofulaceum.

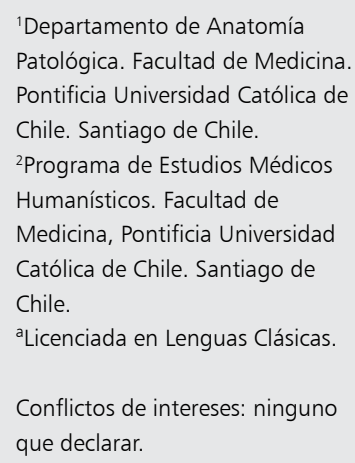

Recibido el 26 de noviembre de 2015, aceptado el 12 de diciembre de 2015 .

Correspondencia a: Dr. Ignacio Duarte Programa de Estudios Médicos Humanísticos. Facultad de Medicina, Pontificia Universidad Católica de Chile. Lira 61, $2^{\circ}$ piso, Santiago, Chile. Teléfono: 223546472 igduarte@gmail.com
$\mathrm{E}$ n el pasado se aplicó el nombre de "escrófula" al aumento de volumen crónico que comprometía principalmente a ganglios linfáticos del cuello. Esta afección ha concitado en diversas épocas el interés de la medicina debido a su frecuencia, las hipótesis sobre sus causas y su evolución aparentemente caprichosa, que oscilaba desde su curación espontánea, sus complicaciones locales, su curación milagrosa hasta su asociación con una enfermedad generalizada y mortal. A fines del siglo XIX, se reconoció su etiología tuberculosa, demostrándose en la centuria siguiente que puede ser causada también por otros bacilos del género Mycobacterium.

El objeto de este trabajo fue indagar acerca de la evolución de las ideas sobre la naturaleza, patoge- nia y tratamientos de la enfermedad escrofulosa en la medicina occidental, desde la medicina clásica griega hasta fines del siglo XVIII.

\section{Cuello porcinoide, glándulas esponjosas y flema}

Para designar tumefacciones glandulares preferentemente cervicales, se utilizó antiguamente en griego el sustantivo femenino choiráde ${ }^{1}$, que significa escollo, peña, relacionado con choirós, que significa puerca. En la literatura médica latina clásica se tradujo como scrofae, que designaba a una puerca paridera y más tarde se incorporaría su forma diminutiva scrofulae ${ }^{2}$. Sin embargo, el 
término más usado era struma, derivado de struo (apilar, amontonar). Otra palabra asociada era glans (bellota) y su diminutivo glándula al referirse a tumefacciones de la garganta, cuello y región inguinal ${ }^{3}$.

En el ámbito de la medicina griega clásica, el libro Perí ton adénon oulomelies, incluido en el Corpus hippocraticum, trata de los ganglios linfáticos. Los describe como constituidos por sustancia esponjosa y friable, que al tacto parece de lana. Están presentes en gran número y tamaño en las partes huecas del cuerpo, las articulaciones, en regiones ricas en sangre y en otras partes húmedas. Se les atribuye la función de drenar y asumir la humedad procedente de aquellas zonas y la que se origina en los esfuerzos físicos. Habitualmente, la cabeza se llena de humedad y de vapores que recibe del resto del cuerpo. Si la cabeza relaja su atracción, se produce flujo a las glándulas: si el flujo es escaso, pueden controlarlo, pero cuando es copioso y morboso, con carácter de flema, se agrandan las glándulas, sobreviene fiebre y se forma la escrófula, que luego puede sufrir erupción ${ }^{4}$.

En el segundo libro de las predicciones del Corpus hippocraticum se afirma que los niños son los que más sufren de tumefacciones escrofulosas, las que fácilmente curan en forma espontánea ${ }^{5}$.

En el siglo I d.C., Celso comenta que el carácter grave y tenaz de las enfermedades escrofulosas explica la multiplicidad de remedios que se proponen, con diversos resultados. Un ungüento de los que menciona consiste en una pomada de lirio, a la que se incorporan los siguientes elementos machacados: resinas, pánace, amoníaco, bedelio, cera, sebo de toro, romero y pimienta ${ }^{6}$.

Galeno, en el siglo II d.C., pensaba que la afección puede originarse en una úlcera que se produce en un enfermo pletórico, la cual se convierte después en un bubón y este en una afección escirrosa "que llaman escrófula". Cuando esta última afección se hace estable puede ser considerada por algunos como primaria, es decir, como si hubiera aparecido por idiopatía, en circunstancia de que se desarrolló por simpatía con la afección inicial. En relación con el tratamiento quirúrgico, relata el caso de un médico ignorante y rústico que al amputar masas escrofulosas cervicales, separó con las uñas los elementos anatómicos, desgarrando los nervios recurrentes: de esta forma curó al niño de sus tumoraciones, pero lo dejó mudo ${ }^{7}$.

En los albores de la Edad Media, el escritor médico Cassius Felix explicaba en el siglo V lo siguiente:

"Las escrófulas son cuerpos redondeados implantados en los tendones, arterias, venas y membranas musculares [...] Cuando se desarrollan en las áreas glandulares a ambos lados de la garganta duelen, y sobresalen como el cuello abultado de una cerda: por eso se llaman scrofae. Son difíciles de curar. Se originan en formaciones ocupadas por un humor malsano. Hay también otra clase de escrófulas, no muy adheridas a la piel, como flotando. Se multiplican como cerdos: por eso, nuevamente, se llaman scrofae. Como dijimos, se originan en regiones glandulares como el cuello, la axila y la ingle".

La monja renana Hildegard von Bingen, en el siglo XII, nombra veintiséis productos para tratar la escrófula. Diez son de origen vegetal, quince de origen animal y uno consiste en hielo fósil. Algunos se aplican pulverizados formando una cataplasma o un ungüento con grasa; otros se administran oralmente crudos o en puré. Recomienda algunos sólo para tratar escrófulas intactas, como la lechuga espinosa que se unta con miel. Otros, exclusivamente para tratar escrófulas fistulizadas, como el ungüento preparado con el cuerpo pulverizado de un estornino ${ }^{8}$.

La obra Regimen Sanitatis atribuida a la escuela Médica de Salerno en el siglo XII, sentencia que " $e l$ higo en cataplasma deshace la escrófula, los tumores $y$ las tumefacciones ganglionares".

El médico británico Gilbertus Anglicus, en el siglo XIII, afirma que las escrófulas “...suelen ser la causa de la sarna, tiña, gangrena, cáncer, fístula, etc." ${ }^{10}$. Como tratamiento aconseja favorecer la supuración, generalmente con emolientes o cataplasmas. Cuando se ablanden pueden abrirse con la lanceta y dejar escapar el pus gradualmente. Como el proceso es tedioso, prefiere la remoción total de las glándulas con el cuchillo, siempre procurando no cortar una glándula que no pueda ser bien aprehendida con la mano y traccionada desde su base. Por su parte, John of Gaddesdenn, médico inglés en el siglo XIV, da una larga lista de remedios, que incluye un método local en base a caracoles y regaliz ${ }^{11}$.

Como opción válida de tratamiento, ambos autores ingleses recomiendan llevar al enfermo ante la presencia del rey, porque aducen que la escrófula se conoce también como morbus regius o "mal del rey", porque el monarca puede curarla tocando al enfermo. 


\section{La ceremonia del toque del rey}

La pretensión de que los reyes de Francia e Inglaterra tenían el poder divino hereditario de curar la escrófula mediante el tacto y la oración dio origen a la ceremonia del "toque del rey". El rito se inició en el siglo XI y se siguió celebrando con cierta regularidad, reuniendo a decenas o cientos de personas cada vez, que acudían con la esperanza de curación.

La difusión de la creencia en ese poder milagroso se basaba en que el monarca, por el hecho de ser ungido y coronado en una ceremonia religiosa, asumía un carácter sacerdotal junto con el poder temporal ${ }^{12}$. Los monarcas de Francia e Inglaterra habrían aprovechado y estimulado la credulidad de sus súbditos para afianzar su poder frente al de los señores feudales y como muestra de preocupación por la salud del pueblo.

La elección de la escrófula puede haberse debido a que parece haber sido muy frecuente, que a veces presentaba episodios de aparente curación, o bien puede confundirse con otros aumentos de volumen cervicales benignos con tendencia espontánea a la regresión.

La supuesta efectividad del "toque del rey" empezó paulatinamente a ser objeto de escepticismo, duda y descrédito, que determinaron la decadencia del rito y su extinción a principios del siglo $\mathrm{XIX}^{13}$.

En 1609, André Du Laurens, médico de Enrique IV de Francia, proponía como causa de la escrófula el beber agua contaminada. Recetaba mojar una pluma en una mezcla de agua, vinagre y miel e introducirla en la garganta para remover el humor malsano acumulado en el cerebro, o también expulsarlo con eméticos y purgantes. Sin embargo, dado que la lesión era resistente a la cirugía o a los fármacos, porque los abscesos reaparecen y proliferan inclusive en las cicatrices, debía recurrirse al poder milagroso del rey ${ }^{14}$.

\section{Afectación multiorgánica, consunción y materia caseosa}

El cirujano Richard Wiseman, que en el siglo XVII acompañó en las ceremonias al rey Carlos II de Inglaterra, escribió un "Tratado del mal del rey”. Definió la escrófula como un tumor originado por acidez del suero de la sangre, que en los sitios afectados se coagula y endurece. La lesión compromete principalmente las glándulas conglobadas, es decir, las que sirven a los conductos linfáticos; por eso muchos autores generalmente confinaban el mal a esos órganos; pero también puede comprometer músculos, membranas, vísceras, tendones o huesos. Se hinchan las glándulas conglobadas del cuello, visibles en los lados del músculo mastoideo hasta abajo a nivel de las clavículas, desde donde puede extenderse al tórax, tanto a la columna vertebral como frecuentemente al pulmón. También pueden afectarse las glándulas conglobadas de axila e ingle y, como se ha comprobado en autopsias, comprometer ganglios linfáticos mesentéricos, aun cuando no haya tumefacción de los ganglios superficiales. Tanto en los ganglios como en las vísceras se encuentran masas que califica de "estrumosas", especialmente en hígado, pulmones y bazo ${ }^{15}$.

El concepto de Wiseman acerca de que la escrófula puede ser una afección local en el cuello, pero también presentarse como una enfermedad diseminada grave, viene a concordar con un relato de 1657, que expone el caso de un paciente que quedó curado de su escrófula por el "toque del rey", pero después falleció de "otro mal, víctima de una lenta consunción"12. Por otra parte, en la ciudad francesa de Reims, una joven llamada Marguerite Rousselet quedó impactada por el abandono de los escrofulosos "víctimas de una enfermedad repugnante, generalmente reputada como incurable y excluidos del Hotel Dieu, escaso de espacio, y por temor a que contagiaran a otros enfermos"16.

Hacia 1645, Marguerite se dedicó a acoger a estos enfermos en una casa particular. Consiguió financiamiento y reclutó voluntarias para cuidarlos. A medida que esta institución crecía pasó a considerarse como sucursal del hospital Hotel Dieu. Fue conocida como Hospicio de los Incurables y después como Hôpital de Saint Marcoul ${ }^{17}$.

En 1689, el médico inglés Richard Morton, en su libro Phthisiología, definía las tisis o consunciones como la mengua de las partes musculares del cuerpo, debida a una sustracción o colicuación de los humores. Las clasificaba en originales y sintomáticas. Las originales no eran efecto de enfermedades preexistentes: entre ellas mencionaba la consunción pulmonar, la consunción nerviosa y la consunción por falta de apetito o de asimilación de los alimentos. Concebía las consunciones sintomáticas como dependencias mediatas de alguna enfermedad precedente. 
Morton explicaba la consunción pulmonar como la consecuencia de rellenamiento, tumefacciones, inflamación o ulceración en ese órgano, lo que conducía al enfermo a la emaciación extrema. Entre las consunciones sintomáticas describía, en primer lugar por su frecuencia, la consunción escrofulosa, provocada por una anormal acrimonia de la sangre que le impide unirse con el quilo, por lo cual estas sustancias se acumulan en los lugares aptos para hincharse y endurecerse más que otros: esto ocurría en primer lugar en el pulmón y luego en las partes glandulosas, dado que ambos sitios tienen una estructura esponjosa. El signo más útil para diagnosticar este mal es la tumefacción de las glándulas de la superficie del cuerpo, y el hallazgo de una oftalmía. Aclaraba que a veces se afectan con este humor solamente las glándulas del pulmón, sin comprometer otras partes. Indica que tanto los tubérculos del pulmón, como las tumefacciones escrofulosas de otras partes son crudas o flegmáticas, y cuando esa materia se cuece, se endurece como tiza o substancia esteatomatosa o, más frecuentemente, se transforma en un material melisérico que se inflama, supura y ulcera dando como resultado un quiste. Acerca de la evolución de la enfermedad, sostiene que algunos pacientes con hinchazones crudas viven enfermizos con tos continua, pero sin fiebre y viven miserablemente, pero si siguen las instrucciones médicas pueden ser liberados del estado escrofuloso y consuntivo. Cuando las lesiones son más cálidas y sufren inflamación y ulceración, esta consunción escrofulosa es muy aguda y el paciente dura pocos meses. A su vez, las hinchazones de naturaleza mediana, susceptibles de inflamarse y evolucionar a la formación de un material como tiza o una sustancia como miel, la consunción no es tan grave, se hace crónica y dura muchos años con una tos continua moderada $^{18}$.

En el siglo XVIII se mencionaban como tratamientos alternativos de la escrófula amuletos o remedios naturales poseedores de una virtud oculta, como la aplicación local de ciertas raíces, semillas, uña de asno quemada, cenizas de cucaracha, mezcladas con miel, o colocar la piel desprendida en la muda de una serpiente, etc. Desde el punto de vista quirúrgico, se indicaba que cuando las escrófulas se fistulizan espontáneamente, la abertura es muy estrecha y se descarga sólo la materia más líquida. El cirujano debe abrirlas para que se descubra el fondo de la úlcera: esto permite aplicar mejor re- medios tópicos y también aislar el ganglio afectado para ligarlo y extirparlo sin dañar partes vecinas ${ }^{19}$.

A fines del siglo XVIII, el médico escocés Mathew Baillie, en su libro de anatomía patológica, se refiere a las "tumefacciones escrofulosas". Manifiesta que las glándulas absorbentes están aumentadas de tamaño y pueden palparse algo más blandas que lo normal. Al corte, a veces exhiben una estructura muy parecida a la normal; pero es más común encontrar que algunas de ellas contienen una materia blanca, blanda, caseosa, mezclada con pus espeso ${ }^{20}$.

\section{Comentario}

El abultamiento de los ganglios cervicales llamado "escrófula" se estimaba muy frecuente, especialmente considerando la cantidad de personas que acudían a ser tocadas por los reyes. Aunque bajo ese nombre puede haberse incluido una variedad de lesiones congénitas o adquiridas como inflamaciones, hiperplasia linfoide, neoplasias o quistes, se reconocía como una sola enfermedad. Las antiguas ideas patogénicas se fundaban en la teoría humoralista hipocrática, según la cual el predominio de uno de los cuatro humores del cuerpo determinaba la mala mezcla de ellos o discrasia, causante de enfermedades. En el caso de la escrófula, el humor redundante (flema, flegma o pituita) se acumulaba principalmente en los ganglios linfáticos del cuello, cercanos a la cabeza.

La concepción humoralista de la escrófula persistió durante mucho tiempo. En el siglo XVII, en consonancia con las ideas iatroquímicas de la época, Wiseman y Morton agregaron la participación de la acidez o acrimonia de la sangre que induce el endurecimiento o coagulación del fluido en los sitios esponjosos, persistiendo Morton en el concepto hipocrático de que el humor malsano acumulado pasaba por una fase de "crudeza" y podía evolucionar a un estado de "cocción" 21 , tornándose en una sustancia esteatomatosa o como tiza, que podía después reblandecerse.

Se empezaba a configurar una relación entre escrófula, tisis y contagio, tanto que se ha considerado que el hospicio de Reims constituye cronológicamente el primer ejemplo de un recinto destinado exclusivamente a aislar y cuidar a pacientes tuberculosos ${ }^{22}$.

Desde el punto de vista morfológico macroscó- 
pico, llama también la atención la descripción de "materia estrumosa" en vísceras de los escrofulosos, unida a los hallazgos de Sylvius en autopsias, en que encontró similitud entre los "tubérculos" pulmonares de la tisis y el aspecto de las glándulas conglobadas tumefactas del cuello y mesenterio. Sylvius recogió la idea de Galeno sobre la existencia de glándulas normalmente invisibles en los pulmones $^{23}$, y les atribuyó ser el asiento de los que llamaba tubérculos ${ }^{24}$. En el siglo XVIII el calificativo de "caseosa" a la materia que reemplazaba la estructura pulmonar y ganglionar en la tisis y en la escrófula agregaba otro elemento común entre ambas afecciones.

Hubo que esperar los avances científicos de los siglos XIX y XX para definir a qué entidad o entidades morbosas correspondía la escrófula.

\section{Referencias}

1. Potter P. Index of symptoms and diseases. En: Hippocrates VI. London: Harvard University Press; 1988. p. 337.

2. Blánquez A. Diccionario Latino-Español. Barcelona: Editorial Sopena; 1954. S.v. scrofula.

3. Barlow F. The King's Evil. Engl Hist Rev 1980; 95: 3-27.

4. Potter P. Hippocrates. VIII. Glands. London, Great Britain: Harvard University Press; 1995. p. 103-25.

5. Potter P. Hippocrates. VIII. Prorrhetic II. P247. London, Great Britain: Harvard University Press; 1995. p. 247.

6. Celso AC. Los ocho libros de medicina. Vol II, Libro V. Barcelona, Editorial Iberia; 1966. p. 15-16.

7. Galeno. Sobre la localización de las enfermedades, Libro I. Madrid, Editorial Gredos S. A.; 1997. p. 146-163.

8. Throop P. Hildegard von Bingen's Phisica. Rochester, Vermont: Healing Arts Press; 1998.

9. Régimen sanitario de Salerno. Traducido del latín por Claudia Chuaqui. An Hist Med 2008; 18: 215-27.

10. Handerson HE. Gilbertus Anglicus Medicine of the thirteenth century. Cleveland, Ohio: The Cleveland Medical
Library Association; 1918. Versión Digital. Google libros.

11. Cholmeley HP. John of Gaddesden and the Rosa Medicinae. Oxford: Oxford Clarendon Press; 1912.

12. Bloch, M. Los reyes taumaturgos. México: Fondo de Cultura Económica; 1988.p. 175-241.

13. Duarte I. La pretendida curación de la escrófula por el toque del rey. Rev Chilena Infectol 2014; 31 (4): 459-67.

14. Tainmont J. The King's evil: scrofula, physicians and the royal touch. B-ENT 2010; 6: 153-9.

15. Wiseman R. Eight chirurgical treatises. Treatise IV: Of the King's evil. London; 1734. 6a Ed. p. 392-512. Versión digital, Google libros.

16. Jadart H. L'Hopital Saint-Marcoul de Reims (16451900); notes et documents pour servir á son histoire et á sa description. Reims: Imprimerie de L'Academie; 1902. Digitalización 2009, Biblioteca Nacional de Francia.

17. Thuret JP. Histoire des lieux de cultes a Reims. lieuxde-culte-reims.blogspot.com/2013/04/ho-183-maisonde-charite-ou-hopital.html (Consultado el 17 de marzo de 2014).

18. Morton R. Phthisiologia: a treatise of consumptions. London: W. and. J. Innis; 1720. Traducido del original, 2a Ed. p. 1-3 y 194-201.Versión digital, Google libros.

19. Violette du Bois, P. Traité des scrofules ou tumeurs blanches, des cancers et loupes, Paris: Paulus de Mesnil; 1726. p. 3-103. Versión digital, Google libros.

20. Baillie M. The morbid anatomy of some of the most important parts of the human body. London. Bulmer and Co.; 1812. 4a Ed. p. 104-111.

21. Chuaqui B. Breve historia de la medicina. Santiago, Chile: Ediciones Universidad Católica de Chile; 2001. p. 37.

22. Quecedo R. La ciudad de la salud: los sanatorios antituberculosos. Ciudades 2011; 14: 213-32.

23. Trail RR. Richard Morton (1637-1698). Med Hist 1970; 14: 166-74.

24. Deleboe F. Sylvius. Ópera Médica, Geneva; de Tournes, 1680. En: Major RH. Classic descriptions of disease. Springfield: Charles C. Thomas, 1982. 3a Ed.p. 526. 